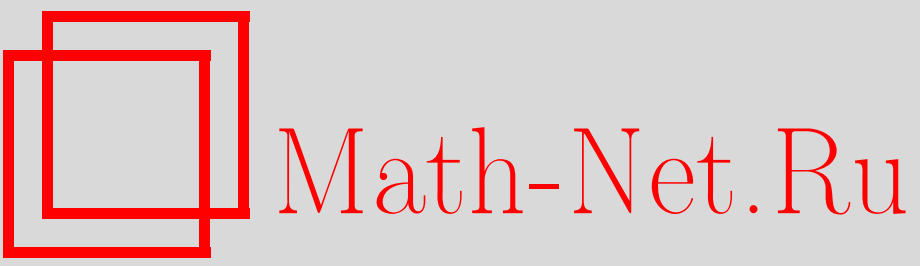

В. И. Усков, Исследование сингулярных возмущений в дескрипторных уравнениях в частных производных первого порядка с матрично-дифференциальным оператором, Итоги науки и техн. Сер. Соврем. мат. и ее прил. Темат. обз., 2019, том $173,132-139$

DOI: https://doi.org/10.36535/0233-6723-2019-173-132-139

Использование Общероссийского математического портала Math-Net.Ru подразумевает, что вы прочитали и согласны с пользовательским соглашением

http: //www.mathnet.ru/rus/agreement

Параметры загрузки:

IP : 3.85 .183 .62

26 апреля 2023 г., 08:39:56 


\title{
ИССЛЕДОВАНИЕ СИНГУЛЯРНЫХ ВОЗМУЩЕНИЙ \\ В ДЕСКРИПТОРНЫХ УРАВНЕНИЯХ В ЧАСТНЫХ ПРОИЗВОДНЫХ ПЕРВОГО ПОРЯДКА С МАТРИЧНО-ДИФФЕРЕНЦИАЛЬНЫМ OПЕРАТОРОМ
}

\author{
(c) 2019 г. $\quad$ В. И. УСКОВ
}

\begin{abstract}
АннотАция. В работе рассматриваются дифференциальные уравнения, вырожденность которых обусловлена наличием матрично-дифференциального операторного коэффициента при производной, возмущенного с помощью малого параметра. Свойства этого оператора применяются при исследовании начально-краевых задач для рассматриваемых уравнений на наличие явления погранслоя; определяются условия регулярности вырождения.
\end{abstract}

Ключевые слова: банахово пространство, матрично-дифференциальный оператор, дифференциальное уравнение первого порядка, малое возмущение, параметр, явление погранслоя.

\section{SINGULAR PERTURBATIONS IN FIRST-ORDER PARTIAL DIFFERENTIAL EQUATIONS WITH MATRIX-DIFFERENTIAL OPERATORS}

\author{
(c) 2019 V. I. USKOV
}

\begin{abstract}
In this paper, we consider differential equations whose degeneracy is due to the presence of a matrix-differential operator coefficient of the derivative perturbed by a small parameter. Properties of this operator are used in the study of initial-boundary-value problems for equations considered for the presence of a boundary layer. Regularity conditions of degeneracy are determined.
\end{abstract}

Keywords and phrases: Banach space, matrix-differential operator, first-order differential equation, small perturbation, parameter, boundary layer.

AMS Subject Classification: 35F40

1. Введение. Рассмотрим начально-краевые задачи для уравнений

$$
\begin{aligned}
& (A+\varepsilon B) \frac{\partial u}{\partial t}=C u(x, t, \varepsilon), \\
& A \frac{\partial u}{\partial t}=(B+\varepsilon C) u(x, t, \varepsilon),
\end{aligned}
$$

где $A, B, C$ - замкнутые линейные операторы, действующие из банахова пространства $E_{1}$ в банахово пространство $E_{2}$ с всюду плотными в $E_{1}$ областями определения; оператор $A$ фредгольмов с нулевым индексом $\left(\Phi_{0}\right.$-оператор); $\varepsilon \in\left(0, \varepsilon_{0}\right) ;(x, t) \in \Omega=[0,2 \pi] \times[0, T]$. 
Впервые уравнения, неразрешенные относительно производной, изучались, по-видимому, в работе А. Пуанкаре [12]. Такие уравнения называют дифберенииально-алгебраическими, или дескрипторными. Исследование таких уравнений сосредоточено в нескольких математических школах: воронежская (С. Г. Крейн, А. Г. Баскаков, С. П. Зубова и др.), челябинская (Г. А. Свиридюк, В. Е. Федоров и их ученики), иркутская (Ю. Е. Бояринцев, Н. А. Сидоров, А. А. Щеглова, В. Ф. Чистяков, М. В. Фалалеев и их ученики), екатеринбургская (И. В. Мельникова и ее ученики). За рубежом активные исследования ведут A. Favini, A. Yagi, S. Campbell, P. Kunkel, V. Mehrmann, R. März и др.

Вырожденные системы уравнений в частных уравнений встречаются в различных областях приложений: гидродинамике (уравнения Навье-Стокса), теплотехнике, электротехнике и т. д. Такие уравнения относят к уравнениям соболевского типа (см. [2]).

Задача Коши для дескрипторных уравнений с $\Phi_{0}$-оператором при производной, возмущенным с помощью малого параметра, исследована на жесткость в $[4,5]$; построено асимптотическое разложение решения (см. [7]).

Несмотря на то, что параметр $\varepsilon$ входит регулярно в уравнения (в пределе тип уравнения не меняется), могут возникать особенности при $\varepsilon \rightarrow 0$.

Целью настоящей работы является применение свойств оператора $A$ при исследовании поставленных задач на наличие явления погранслоя; определение условий регулярности вырождения.

2. Необходимые сведения. Приведем сведения, необходимые для решения поставленных задач.

Свойство 1. Пусть линейный оператор $A$ действует из банахова пространства $E_{1}$ в банахово пространство $E_{2}$. Свойство $\Phi_{0}$ этого оператора влечет разложения

$$
E_{1}=\operatorname{Coim} A \oplus \operatorname{Ker} A, \quad E_{2}=\operatorname{Im} A \oplus \operatorname{Coker} A,
$$

здесь $\operatorname{Coim} A-$ прямое дополнение к ядру $\operatorname{Ker} A ; \operatorname{Coker} A-$ дефектное подпространство; $\operatorname{dim} \operatorname{Ker} A=\operatorname{dim}$ Coker $A<\infty$; сужение $\tilde{A}$ оператора $A$ на $\operatorname{Coim} A$ имеет ограниченный обратный $\tilde{A}^{-1}$ (см. [9]).

Пусть $D$ - некоторый линейный ограниченный оператор, $\omega$ - тип полугруппы этого оператора. Имеет место следующая оценка (см. [8]):

$$
\|\exp (t D)\| \leqslant \mu \exp (\omega t), \quad \mu=\text { const }>0 .
$$

2.1. Поведение решения уравнения с малым параметром. Рассмотрим допредельное уравнение с искомой функцией $z$ и малым параметром $\varepsilon \in\left(0, \varepsilon_{0}\right)$ :

$$
\Phi(\varepsilon, t, z(t, \varepsilon))=0
$$

и предельное уравнение для уравнения (5):

$$
\bar{\Phi}(t, \bar{z}(t))=0 .
$$

Возможно следующее поведение решения $z(t, \varepsilon)$ при $\varepsilon \rightarrow 0$ :

(a) $z(t, \varepsilon) \Rightarrow \bar{z}(t)$ (равномерная сходимость);

(b) $z(t, \varepsilon)=\bar{z}(t)+v(t, \varepsilon)$, где $v(t, \varepsilon)$ - функция погранслоя вблизи точки $t=0$ (явление погранслоя);

(c) остальные случаи: $\|z(t, \varepsilon)\| \rightarrow \infty$ или $z(t, \varepsilon)$ не имеет предела.

Ограниченная функция $v(t, \varepsilon)$, определенная на $[0, T]$, называется функиией погранслоя вблизи точки $t=0$, если при $\varepsilon \rightarrow 0$ имеет место соотношение $v(t, \varepsilon) \Rightarrow 0$ на $\left[t^{\prime}, T\right]$ для всех $t^{\prime} \in(0, T)$ и $v(t, \varepsilon) \nRightarrow 0$ на $[0, T]$ (см. [4]). 
2.2. O матрично-дифференциальном операторе. Рассмотрим матрично-дифференциальный оператор

$$
A=\left(\begin{array}{cc}
\frac{\partial}{\partial x} & -1 \\
1 & \frac{\partial}{\partial x}
\end{array}\right)
$$

с областью определения

$$
\operatorname{dom} A=\left\{\left(\begin{array}{l}
v_{1}(x) \\
v_{2}(x)
\end{array}\right), v_{k}(x) \in C^{2}[0,2 \pi], v_{k}(0)=v_{k}(2 \pi), k=1,2\right\},
$$

действующий в банаховом пространстве

$$
E=\left\{\left(\begin{array}{l}
v_{1}(x) \\
v_{2}(x)
\end{array}\right), v_{k}(x) \in C[0,2 \pi], k=1,2\right\} .
$$

В [6] доказано наличие $\Phi_{0}$-свойства у оператора $A$ (разложения (3)), где

Ker $A=\left\{c_{1} e_{1}(x)+c_{2} e_{2}(x), c_{1}, c_{2} \in \mathbb{C}\right\}, \quad$ Coker $A=\left\{k_{1} e_{1}(x)+k_{2} e_{2}(x), k_{1}, k_{2} \in \mathbb{C}\right\}$,

$$
e_{1}(x)=\left(\begin{array}{r}
\cos x \\
-\sin x
\end{array}\right), \quad e_{2}(x)=\left(\begin{array}{c}
\sin x \\
\cos x
\end{array}\right) .
$$

Проекторы $P(x), Q(x)$ на $\operatorname{Ker} A$, Coker $A$ соответственно равны

$$
P(x)=Q(x)=\left(\begin{array}{cc}
\frac{1}{2 \pi} \int_{0}^{2 \pi}(\cdot) \cos (x-s) d s & \frac{1}{2 \pi} \int_{0}^{2 \pi}(\cdot) \sin (x-s) d s \\
-\frac{1}{2 \pi} \int_{0}^{2 \pi}(\cdot) \sin (x-s) d s & \frac{1}{2 \pi} \int_{0}^{2 \pi}(\cdot) \cos (x-s) d s
\end{array}\right) ;
$$

полуобратный оператор $A^{-}(x)=\left(H_{i j}\right), i, j=1,2$, где

$$
\begin{aligned}
& H_{11}=-\int_{x}^{2 \pi}(\cdot) \cos (x-s) d s+\frac{1}{2 \pi} \int_{0}^{2 \pi}(\cdot) \cdot s \cdot \cos (x-s) d s, \\
& H_{12}=-\int_{0}^{2 \pi}(\cdot) \sin (x-s) d s+\frac{1}{2 \pi} \int_{0}^{2 \pi}(\cdot) \cdot s \cdot \sin (x-s) d s, \\
& H_{21}=\int_{0}^{2 \pi}(\cdot) \sin (x-s) d s-\frac{1}{2 \pi} \int_{0}^{2 \pi}(\cdot) \cdot s \cdot \sin (x-s) d s, \\
& H_{22}=-\int_{0}^{2 \pi}(\cdot) \cos (x-s) d s+\frac{1}{2 \pi} \int_{0}^{2 \pi}(\cdot) \cdot s \cdot \cos (x-s) d s,
\end{aligned}
$$

Оператор $A_{1}=Q P=P$ обратим; $A_{1}^{-1}=I$ ( $I$ - единичный оператор в соответствующем подпространстве).

Нетрудно видеть, что операторы $Q(x), P(x)$ и $A^{-}(x)$ ограничены при каждом $x \in[0,2 \pi]$.

2.3. Решение задачи Коши для дескрипторного уравнения. Рассмотрим задачу

$$
(A+\varepsilon B) \frac{d z}{d t}=C z(t, \varepsilon), \quad z(0, \varepsilon)=z^{0}(\varepsilon) \in E_{1},
$$

где $A, B, C$-замкнутые линейные операторы, действующие из банахова пространства $E_{1}$ в банахово пространство $E_{2}$ с всюду плотными в $E_{1}$ областями определения; $A-\Phi_{0}$-оператор; $z^{0}(\varepsilon)-$ голоморфная в окрестности точки $\varepsilon=0$ функция; $z(t, \varepsilon) \in E_{1} ; t \in[0, T] ; \varepsilon \in\left(0, \varepsilon_{0}\right)$. 
В случае обратимости ненулевого оператора

$$
A_{1}^{(1)}=Q B P: \operatorname{Ker} A \rightarrow \operatorname{Coker} A
$$

применив обозначения

$$
\begin{gathered}
S_{0}^{(1)}=Q B, \quad T_{0}^{(1)}=A^{-} B, \quad R_{0}=\left(I+\varepsilon T_{0}^{(1)}\right)^{-1}, \\
K_{0}=I-\left(A_{1}^{(1)}\right)^{-1} S_{0}^{(1)} R_{0}, \quad V_{1}(\varepsilon)=R_{0} K_{0} A^{-} C+\varepsilon^{-1} R_{0}\left(A_{1}^{(1)}\right)^{-1} Q C,
\end{gathered}
$$

сформулируем следующий результат.

Лемма 1 (см. [3]). Пусть оператор $V_{1}(\varepsilon): \operatorname{Coim} A \rightarrow \operatorname{Coim} A$, определяемый формулой (10), ограничен и сильно непрерывен при каждом $\varepsilon \in\left(0, \varepsilon_{0}\right)$. Тогда решение задачи (9) существует при выполнении условия

$$
0<\varepsilon\left\|T_{0}^{(1)}\right\|<1 .
$$

Это решение единственно и имеет вид

$$
z(t, \varepsilon)=\exp \left(t \cdot V_{1}(\varepsilon)\right) z^{0}(\varepsilon)
$$

Теперь рассмотрим задачу

$$
A \frac{d z}{d t}=D z(t), \quad z(0)=z^{0} \in E_{1},
$$

где $A, D$ - замкнутые линейные операторы, действующие из банахова пространства $E_{1}$ в банахово пространство $E_{2}$ с всюду плотными в $E_{1}$ областями определения; $A-\Phi_{0}$-оператор; $z(t) \in E_{1}$; $t \in[0, T]$.

Задача (14) решена методом каскадной декомпозиции, модифицированным в [10]. В случае обратимости ненулевого оператора

$$
A_{1}^{(2)}=Q D P: \operatorname{Ker} A \rightarrow \operatorname{Coker} A,
$$

применив обозначения

$$
S_{0}^{(2)}=Q D, \quad T_{0}^{(2)}=A^{-} D, \quad \hat{S}_{0}=I-\left(A_{1}^{(2)}\right)^{-1} S_{0}, \quad T_{1}=T_{0}^{(2)} \hat{S}_{0},
$$

сформулируем следующий результат.

Лемма 2. Пусть оператор $T_{1}: \operatorname{Coim} A \rightarrow \operatorname{Coim} A$, определяемый формулой (16), ограничен. Тогда решение задачи (14) существует при выполнении условия

$$
Q D z^{0}=0 .
$$

Это решение единственно, имеет вид

$$
z(t, \varepsilon)=\hat{S}_{0} \exp \left(t \cdot T_{1}\right)(I-P) z^{0}
$$

и обладает следуюшим свойством:

$$
Q D z(t) \equiv 0, \quad t \in[0, T] .
$$

3. Начально-краевая задача для дескрипторного уравнения в частных производных первого порядка с возмущением слева. Рассмотрим задачу

$$
(A+\varepsilon B) \frac{\partial u}{\partial t}=C u(x, t, \varepsilon), \quad u(x, 0, \varepsilon)=u^{0}(x, \varepsilon), \quad u(0, t, \varepsilon)=u(2 \pi, t, \varepsilon),
$$

где оператор $A$ определяется формулами (6)-(8); операторы $B, C$ задаются вещественными числовыми матрицами $B=\left(b_{i j}\right), C=\left(c_{i j}\right) ; u^{0}(x, \varepsilon)$ - достаточно гладкая по $x$ и голоморфная в окрестности точки $\varepsilon=0$ функция; $\varepsilon \in\left(0, \varepsilon_{0}\right) ;(x, t) \in \Omega=[0,2 \pi] \times[0, T]$.

Под решением задачи (20) подразумевается функция $u(x, t, \varepsilon)$, обладающая следующими свойствами:

(i) $u(x, t, \varepsilon)$ дифференцируема по $t$ и дважды по $x$;

(ii) $u(x, t, \varepsilon)$ непрерывна по $x$ вместе со своими производными $\partial u / \partial x, \partial^{2} u / \partial x^{2}$; 
(iii) $u(x, t, \varepsilon)$ удовлетворяет (20) при всех $(x, t) \in \Omega, \varepsilon \in\left(0, \varepsilon_{0}\right)$.

Задача (20) - это задача вида (9). Предельная задача имеет вид

$$
A \frac{\partial \bar{u}}{\partial t}=C \bar{u}(x, t), \quad \bar{u}(x, 0)=\bar{u}^{0}(x), \quad \bar{u}(0, t)=\bar{u}(2 \pi, t) .
$$

Пусть выполнено одно из неравенств: $b_{11}+b_{12} \neq 0$ или $b_{12}-b_{21} \neq 0$. Рассмотрим случай, когда оператор $A_{1}^{(1)}(x)$, определяемый формулой $(10)$, ненулевой и обратимый в Ker $A$. Пусть при всех $(x, \varepsilon) \in[0,2 \pi] \times\left(0, \varepsilon_{0}\right)$ имеет место неравенство $(12)$.

Здесь и далее используются обозначения: $K^{*}$-присоединенная матрица для матрицы $K$ (см. [1]); $\tilde{B}=B+B^{*}, \tilde{C}=C+C^{*}$.

Вычисления показывают, что

$$
A_{1}^{(1)}(x)=\frac{1}{2} \tilde{B} P(x)
$$

Обратный оператор существует (в подпространстве $\operatorname{Ker} A$ ) при выполнении условия $\operatorname{det} \tilde{B} \neq 0$; решив уравнение

$$
A_{1}^{(1)}(x) \xi(x)=\eta(x), \quad \xi(x) \in \operatorname{Ker} A, \quad \eta(x) \in \operatorname{Coker} A,
$$

имеем:

$$
\left(A_{1}^{(1)}\right)^{-1}=2 \tilde{B}^{-1} .
$$

Оператор $V_{1}$ (см. обозначения (11)) ограничен и сильно непрерывен. Тогда в силу леммы 1 решение задачи (20) единственно и определяется по формуле (13).

Легко видеть, что особенности в поведении решения порождаются экспонентой $\exp \left(t \cdot V_{1}(x, \varepsilon)\right)$. Оператор $V_{1}$ допускает асимптотическое представление

$$
V_{1}(x, \varepsilon)=\varepsilon^{-1} W+o(1) \quad \text { при } \varepsilon \rightarrow 0,
$$

где

$$
W=W(x)=2 \tilde{B}^{-1} Q(x) C ;
$$

добавка o(1) вмещает в себя нормы ограниченных операторов. Отсюда следует равенство

$$
\exp \left(t \cdot V_{1}\right)=\mu(\varepsilon) \exp \left(\frac{t}{\varepsilon} W\right)
$$

где $\mu(\varepsilon)$ - равномерно ограниченная функция. Пользуясь оценкой (4), имеем:

$$
\left\|\exp \left(t \cdot V_{1}\right)\right\| \leqslant \mu^{*} \cdot \exp \left(\omega_{1} \frac{t}{\varepsilon}\right), \quad \mu^{*}=\max |\mu(\varepsilon)|,
$$

где $\omega_{1}$ - тип полугруппы оператора $W$. Из этой оценки следует, что неравенство $\omega_{1}<0$ является условием регулярности вырождения. Таким образом, имеет место следующее поведение решения при $\varepsilon \rightarrow 0$ задачи (20).

Теорема 1. Пусть $W(x)$ - производщий оператор полугруппы отрищательного типа при каждом $x$. Тогда имеет место случай (b), и функции погранслоя иметот переменную $t / \varepsilon$.

Изучим спектр оператора $W(x)$. Введем обозначения $\tilde{B}^{-1}=\left(\hat{b}_{i j}\right)$,

$$
\begin{array}{llll}
\hat{k}_{1}=\frac{\hat{b}_{11} c_{11}+\hat{b}_{12} c_{21}}{\pi}, & \hat{k}_{2}=\frac{\hat{b}_{11} c_{12}+\hat{b}_{12} c_{22}}{\pi}, & \hat{k}_{3}=\frac{\hat{b}_{11} c_{21}-\hat{b}_{12} c_{11}}{\pi}, & \hat{k}_{4}=\frac{\hat{b}_{11} c_{22}-\hat{b}_{12} c_{12}}{\pi}, \\
\hat{k}_{5}=\frac{\hat{b}_{21} c_{11}+\hat{b}_{22} c_{21}}{\pi}, & \hat{k}_{6}=\frac{\hat{b}_{21} c_{12}+\hat{b}_{22} c_{22}}{\pi}, & \hat{k}_{7}=\frac{\hat{b}_{21} c_{21}-\hat{b}_{22} c_{11}}{\pi}, & \hat{k}_{8}=\frac{\hat{b}_{21} c_{22}-\hat{b}_{22} c_{12}}{\pi} .
\end{array}
$$


Оператор $W(x)=\left(W_{i j}\right), i, j=1,2$, определяется равенствами

$$
\begin{aligned}
& W_{11}=\hat{k}_{1} \int_{0}^{2 \pi}(\cdot) \cos (x-s) d s+\hat{k}_{3} \int_{0}^{2 \pi}(\cdot) \sin (x-s) d s, \\
& W_{12}=\hat{k}_{2} \int_{0}^{2 \pi}(\cdot) \cos (x-s) d s+\hat{k}_{4} \int_{0}^{2 \pi}(\cdot) \sin (x-s) d s, \\
& W_{21}=\hat{k}_{5} \int_{0}^{2 \pi}(\cdot) \cos (x-s) d s+\hat{k}_{7} \int_{0}^{2 \pi}(\cdot) \sin (x-s) d s, \\
& W_{22}=\hat{k}_{6} \int_{0}^{2 \pi}(\cdot) \cos (x-s) d s+\hat{k}_{8} \int_{0}^{2 \pi}(\cdot) \sin (x-s) d s .
\end{aligned}
$$

Из спектрального уравнения

$$
W(x) v(x)=\lambda v(x), \quad v(x)=\left(\begin{array}{l}
v_{1}(x) \\
v_{2}(x)
\end{array}\right)
$$

следует уравнение для нахождения собственных значений оператора $W$ :

$$
\operatorname{det}\left(\begin{array}{cccc}
\pi \hat{k}_{1}-\lambda & \pi \hat{k}_{2} & -\pi \hat{k}_{3} & -\pi \hat{k}_{4} \\
\pi \hat{k}_{5} & \pi \hat{k}_{6}-\lambda & -\pi \hat{k}_{7} & -\pi \hat{k}_{8} \\
\pi \hat{k}_{3} & \pi \hat{k}_{4} & \pi \hat{k}_{1}-\lambda & \pi \hat{k}_{2} \\
\pi \hat{k}_{7} & \pi \hat{k}_{8} & \pi \hat{k}_{5} & \pi \hat{k}_{6}-\lambda
\end{array}\right)=0 .
$$

4. Начально-краевая задача для дескрипторного уравнения в частных производных первого порядка с возмущением справа. Рассмотрим задачу

$$
A \frac{\partial u}{\partial t}=(B+\varepsilon C) u(x, t, \varepsilon), \quad u(x, 0, \varepsilon)=u^{0}(x, \varepsilon), \quad u(0, t, \varepsilon)=u(2 \pi, t, \varepsilon),
$$

где оператор $A$ определяется формулами (6)-(8), операторы $B, C$ задаются вещественными числовыми матрицами $B=\left(b_{i j}\right), C=\left(c_{i j}\right) ; u^{0}(x, \varepsilon)$ - достаточно гладкая по $x$ и голоморфная в окрестности точки $\varepsilon=0$ функция; $\varepsilon \in\left(0, \varepsilon_{0}\right) ;(x, t) \in \Omega$.

Под решением задачи $(21)$ подразумевается функция $u(x, t, \varepsilon)$, обладающая следующими свойствами:

(i) $u(x, t, \varepsilon)$ дифференцируема по $t$ и дважды по $x$;

(ii) $u(x, t, \varepsilon)$ непрерывна по $x$ вместе со своими производными $\partial u / \partial x, \partial^{2} u / \partial x^{2}$;

(iii) $u(x, t, \varepsilon)$ удовлетворяет (21) при всех $(x, t) \in \Omega, \varepsilon \in\left(0, \varepsilon_{0}\right)$.

Предельная задача для задачи (21) имеет вид

$$
A \frac{\partial \bar{u}}{\partial t}=B \bar{u}(x, t), \quad \bar{u}(x, 0)=\bar{u}^{0}(x), \quad \bar{u}(0, t)=\bar{u}(2 \pi, t) .
$$

Рассмотрим случай, когда оператор $A_{1}^{(2)}(x, \varepsilon)$, определяемый формулой $(15)$ с $D=B+\varepsilon C$ ненулевой и обратимый в $\operatorname{Ker} A$ при каждом $\varepsilon$. Вычисления показывают, что

$$
A_{1}^{(2)}(x, \varepsilon)=\frac{1}{2} \tilde{D}(\varepsilon) P(x),
$$

где $\tilde{D}(\varepsilon)=\tilde{B}+\varepsilon \tilde{C}$. Обратный оператор существует (в подпространстве $\operatorname{Ker} A$ ) при выполнении условия

$$
\Delta_{\varepsilon} \stackrel{\text { def }}{=} \operatorname{det} D(\varepsilon) \neq 0 \quad \text { при каждом } \varepsilon \text {. }
$$

Решив уравнение

$$
A_{1}^{(2)}(x, \varepsilon) \xi(x)=\eta(x), \quad \xi(x) \in \operatorname{Ker} A, \quad \eta(x) \in \text { Coker } A,
$$


получим

$$
\left(A_{1}^{(2)}\right)^{-1}=2 \tilde{D}^{-1}(\varepsilon)=2 \Delta_{\varepsilon}^{-1} \tilde{D}^{*}(\varepsilon) .
$$

Оператор $T_{1}(x, \varepsilon)$ (см. обозначения $\left.(16)\right)$ ограничен и сильно непрерывен. Поэтому в силу леммы 2 решение задачи (21) существует при выполнении условия (17). Это решение единственно, определяется формулой (18) и обладает свойством (19).

Далее,

$$
\begin{gathered}
T_{1}=A^{-} B+\varepsilon A^{-} C+\Delta_{\varepsilon}^{-1} G(\varepsilon), \\
G(\varepsilon)=G_{0}+\varepsilon G_{1}+o(\varepsilon), \quad G_{0}=-2 A^{-} B \tilde{B}^{*} Q B, \quad G_{1}=-2 A^{-} B\left(\tilde{B}^{*} Q C+\tilde{C}^{*} Q B\right)-2 A^{-} C \tilde{B}^{*} Q B ;
\end{gathered}
$$

добавка $o(\varepsilon)$ вмещает в себя нормы ограниченных операторов. Операторы $G_{0}, G_{1}$ также ограничены.

Нетрудно видеть, что особенности в поведении решения порождаются экспонентой $\exp (t$. $\left.T_{1}(x, \varepsilon)\right)$. Распишем определитель $\Delta_{\varepsilon}$ в виде

$$
\Delta_{\varepsilon}=\Delta_{0}+\varepsilon \Delta_{1}+\varepsilon^{2} \Delta_{2}
$$

где

$$
\Delta_{0}=\operatorname{det} \tilde{B}, \quad \Delta_{1}=\operatorname{det}\left(\begin{array}{ll}
\tilde{b}_{11} & \tilde{b}_{12} \\
\tilde{c}_{21} & \tilde{c}_{22}
\end{array}\right)+\operatorname{det}\left(\begin{array}{ll}
\tilde{c}_{11} & \tilde{c}_{12} \\
\tilde{b}_{21} & \tilde{b}_{22}
\end{array}\right), \quad \Delta_{2}=\operatorname{det} \tilde{C}
$$

$\left(\tilde{b}_{i j}, \tilde{c}_{i j}\right.$ - элементы матриц $\left.\tilde{B}, \tilde{C}, i, j=1,2\right)$. Получаем следующие импликации:

$$
\begin{aligned}
\Delta_{0} \geqslant 0, \quad \Delta_{0}=0 & \Leftrightarrow\left\{\begin{array}{l}
b_{11}+b_{22}=0, \\
b_{12}-b_{21}=0
\end{array} \quad \Leftrightarrow \quad \tilde{B}=0 \quad \Leftrightarrow \quad G_{0}=0 ;\right. \\
\Delta_{2} \geqslant 0, \quad \Delta_{2}=0 & \Leftrightarrow\left\{\begin{array}{l}
c_{11}+c_{22}=0, \\
c_{12}-c_{21}=0
\end{array} \quad \Leftrightarrow \quad \tilde{C}=0 ;\right. \\
\Delta_{0}=0 & \Rightarrow \quad \Delta_{1}=0 .
\end{aligned}
$$

Пусть $\Delta_{0} \neq 0$. В этом случае $T_{1}=O(1)$ при $\varepsilon \rightarrow 0$, что в силу оценки (4) влечет случай (а) поведения решения.

Теперь рассмотрим случай $\Delta_{0}=0$. Из импликаций $(23)$ следует, что $T_{1}=O\left(\varepsilon^{-1}\right)$ при $\varepsilon \rightarrow 0$. Экспонента расписывается в виде

$$
\exp \left(t \cdot T_{1}\right)=\mu(\varepsilon) \exp \left(\frac{t}{\varepsilon} \cdot \frac{G_{1}}{\Delta_{2}}\right)
$$

где $\mu(\varepsilon)$ - равномерно ограниченная функция. Пользуясь оценкой (4), имеем

$$
\left\|\exp \left(t \cdot T_{1}\right)\right\| \leqslant \mu^{*} \cdot \exp \left(\frac{1}{\Delta_{2}} \cdot \frac{t}{\varepsilon} \cdot \omega_{2}\right), \quad \mu^{*}=\max |\mu(\varepsilon)|,
$$

где $\omega_{2}$ - тип полугруппы оператора $G_{1}$. Из последней оценки вытекает, что неравенство $\omega_{2}<0$ является условием регулярности вырождения.

Замечание 1. При $\Delta_{2}=0$ оператор $A_{1}^{(2)}$ необратим, так что требуется дальнейшее исследование.

Таким образом, имеет место следующее поведение решения при $\varepsilon \rightarrow 0$ задачи (21).

Теорема 2. При условии $\Delta_{0}>0$ имеет место случай (а). Если $G_{1}(x)-$ производящий оператор полугруппы отрицательного типа при каждом $x$ и выполнены условия $\Delta_{0}=0, \Delta_{2}>0$, то имеет место случай (b), и функиии погранслоя имеют переменную $t / \varepsilon$. 
Для изучения спектра оператора $G_{1}(x)$ введем обозначения $\tilde{c}_{1}=\tilde{c}_{11}=\tilde{c}_{22}, \tilde{c}_{2}=\tilde{c}_{12}=-\tilde{c}_{21}$,

$$
\begin{aligned}
& \tilde{k}_{1}=\frac{\left(b_{11}-b_{22}\right)\left(b_{21} \tilde{c}_{1}-b_{11} \tilde{c}_{2}\right)-\left(b_{12}+b_{21}\right)\left(b_{11} \tilde{c}_{1}+b_{21} \tilde{c}_{2}\right)}{4 \pi}, \\
& \tilde{k}_{2}=-\frac{\left(b_{11}-b_{22}\right)\left(b_{11} \tilde{c}_{1}+b_{21} \tilde{c}_{2}\right)+\left(b_{12}+b_{21}\right)\left(b_{21} \tilde{c}_{1}-b_{11} \tilde{c}_{2}\right)}{4 \pi}, \\
& \tilde{k}_{3}=\frac{\left(b_{11}-b_{22}\right)\left(b_{22} \tilde{c}_{1}-b_{12} \tilde{c}_{2}\right)-\left(b_{12}+b_{21}\right)\left(b_{12} \tilde{c}_{1}+b_{22} \tilde{c}_{2}\right)}{4 \pi}, \\
& \tilde{k}_{4}=-\frac{\left(b_{11}-b_{22}\right)\left(b_{12} \tilde{c}_{1}+b_{22} \tilde{c}_{2}\right)+\left(b_{12}+b_{21}\right)\left(b_{22} \tilde{c}_{1}-b_{12} \tilde{c}_{2}\right)}{4 \pi} .
\end{aligned}
$$

Из спектрального уравнения

$$
G_{1}(x) v(x)=\lambda v(x), \quad v(x)=\left(\begin{array}{l}
v_{1}(x) \\
v_{2}(x)
\end{array}\right)
$$

вытекает уравнение для нахождения собственных значений оператора $G_{1}$ :

$$
\begin{gathered}
\operatorname{det}\left(\begin{array}{cccc}
\pi \tilde{k}_{1}-\lambda & -\pi \tilde{k}_{2} & \pi \tilde{k}_{3} & -\pi \tilde{k}_{4} \\
\pi \tilde{k}_{2} & \pi \tilde{k}_{1}-\lambda & \pi \tilde{k}_{4} & \pi \tilde{k}_{3} \\
-\pi \tilde{k}_{2} & -\pi \tilde{k}_{1} & -\pi \tilde{k}_{4}-\lambda & -\pi \tilde{k}_{3} \\
\pi \tilde{k}_{1} & -\pi \tilde{k}_{2} & \pi \tilde{k}_{3} & -\pi \tilde{k}_{4}-\lambda
\end{array}\right)=0 . \\
\text { СПИСОк лИТЕРАТУРЫ }
\end{gathered}
$$

\section{СПИСОК ЛИТЕРАТУРЫ}

1. Гантмахер Ф. Р. Теория матриц. - М.: Физматлит, 2004.

2. Демиденко Г. В., Успенский С. В. Уравнения и системы, не разрешенные относительно старшей производной. - Новосибирск: Научная книга, 1998.

3. Зубова С. П. Решение однородной задачи Коши для уравнения с нетеровым оператором при производной// Докл. РАН. - 2009. - 428, № 4. - С. 444-446.

4. Зубова С. П. О роли возмущений в задаче Коши для уравнения с фредгольмовым оператором при производной // Докл. РАН. - 2014. - 454, № 4. - С. 383-386.

5. Зубова С. П., Раецкая Е. В. Исследование жесткости дескрипторной динамической системы в банаховом пространстве// Пробл. мат. анал. - 2015. - 79. - С. 127-132.

6. Зубова С. П., Раецкая Е. В., Усков В. И. О свойствах вырожденности некоторого дифференциального оператора и их применение// Фундам. прикл. мат. (в печати).

7. Зубова С. П., Усков В. И. Асимптотическое решение задачи Коши для уравнения первого порядка с малым параметром в банаховом пространстве. Регулярный случай// Мат. заметки. - 2018. - 103, № 3. - C. 393-404.

8. Крейн С. Г. Линейные дифференциальные уравнения в банаховом пространстве. - М.: Наука, 1967.

9. Никольский C. М. Линейные уравнения в линейных нормированных пространствах// Изв. АН СССР. Сер. мат. - 1943. - 7, № 3. - С. 147-166.

10. Усков В. И. Решение задачи Коши для дескрипторного уравнения первого порядка// Вестн. Воронеж. гос. ун-та. Сер. Физ. Мат. (в печати).

11. Kunkel P., Mehrmann V. Differential-Algebraic Equations: Analysis and Numerical Solutions. — Zurich: EMA, 2006.

12. Poincaré H. Sur l'equilibre d'une masse fluide animee d'un movement de rotation// Acta Math. — 1885. - 7. - P. 259-380.

Усков Владимир Игоревич

Воронежский государственный лесотехнический университет им. Г. Ф. Морозова

E-mail: vum1@yandex.ru 